\title{
MY CONNECTIONS WITH PROF. N. N. BOGOLIUBOV IN THE LABORATORY OF THEORETICAL PHYSICS JINR IN DUBNA
}

\author{
Z. M. Galasiewicz \\ Wroctaw University of Technology, Institute of Physics, ul. Wybrzeże Wyspiańskiego 27, 50-370 Wroctaw, Poland
}

(Received May 1, 1997)

\begin{abstract}
Memoirs about collaboration and meetings with outstanding scientist of our century — Professor N. N. Bogoliubov - are presented.

PACS number(s): 01.65.+g
\end{abstract}

In 1956 I got my Ph. D. in physics at the University of Wroclaw for a dissertation strongly based on Bogoliubov's method of additional collective variables. Just after the establishment of the Joint Institute for Nuclear Research in 1956 prof. J. Rzewuski left our Institute for a one year stay in Dubna. The head of the Laboratory of Theoretical Physics in JINR was the world famous theoretical physicist and mathematician prof. Nikolai N. Bogoliubov. Knowing my deep interest the Bogoliubov's work prof. J. Rzewuski took steps towards organising my visit to the Laboratory of Theoretical Physics after the end of his stay in Dubna. Thanks to his initiative I arrived in Dubna in 1958 to stay there for two years and it was the beginning of the most significant scientific adventure of my life. It was a time just after the discovery of the exciting theory of superconductivity of metals and alloys proposed by Bardeen-Cooper-Schriefer and Bogoliubov.

Prof. N. N. Bogoliubov received me in a very friendly way. He was just preparing to publication an important paper devoted to the problem compensating the "dangerous diagrams" in the method of selfconsistent field [1]. It included a consideration of collective oscillations and their application to the electrodynamics of the superconducting state. Professor suggested that I should study the same problem on the basis of approximate second quantization.

In the course of my investigations I reveald that the spectrum of collective oscillations was divided into two branches: for the pairs of fermions having opposite spins and for the pairs having parallel spins [2]. The equation of compensation of "dangerous diagrams" was considered for the pairs with the zero spin. Besides the results of [1], obtained now on the basis of a totally different approach, I considered also the contribution of excitations with the spin \pm 1 to a superconducting current. The most important result of this paper was an inspiration to consider the equation of "dangerous diagrams" for the pairs with the spin \pm 1 . This led to the first theory of the anisotropic (" $p$ " pairing) superfluid phase (polar) in the fermion system. It was published as Dubna preprint (1959) and in journal [3]. In 1972 the superfluidity of helium 3 was discovered. Later on a more careful analysis showed that it was connected with an anisotropic phase. In connection with this in 1975 , due to the initiative of N. N. Bogoliubov, JINR reedited my two preprints and it was underlined in the Introduction that the theory of
Fermi systems with the " $p$ " pairing was proposed for the first time in JINR. This theory formed the basis of my habilitation thesis in 1961.

My first stay in Dubna lasted two years. At that time, in order to reach the Laboratory of Theoretical Physics situated near the Laboratory of Nuclear Problems, we needed a bus ride or it was necessary to walk a rather long distance.

In 1960-72 I was a member of the Scientific Council of the Laboratory of Theoretical Physics and after the reorganization I represented during a long period of time the Polish side at the meetings of the Council. This gave me an opportunity to visit JINR mostly twice per year and have more frequent contacts with N. N. Bogoliubov. In 1961 and 1963 he published two fundamental papers. In the first one [4] he introduced the notion of quasiaverages. They describe the situation of the system after its transition to the new phase in which some former symmetries have been broken. For this description it is necessary to add to the initial Hamiltonian new (even infinitesimal) terms leading to the breaking of the mentioned symmetries. In the second paper [5] N. N. Bogoliubov derived, on the basis of the microscopic approach, the Landau hydrodynamic equations for superfluid helium 4 without dissipation being considered. He introduced into the Hamiltonian new terms (with "sources of particles") breaking the gauge "symmetry" (invariance) of the Hamiltonian. With the help of the sources it was easier to present the Bogoliubov " $1 / q^{2}$ theorem" for superfluid helium 4 . This theorem shows that the Green functions constructed with the help of the order parameter operator, have the $1 / q^{2}$ singularity. The mentioned theorem plays an essential role in the examination of the long-range ordering in superfluid helium. Using this theorem one can show that the Yang's criterion for the existence of the long-range order (criterion of superfluidity) is not satisfied in one and two dimensions.

In the summer of 1963 during my visit to Dubna N. N. Bogoliubov suddenly invited me to his office. He informed me that there are two parallel problems. Namely, the preparation for the publication of his paper devoted to hydrodynamics of superfluid liquid (more than 40 pages). This meant it was necessary to examine very carefully the manuscript in order to find eventual misprints (and I have found about 60 !). The second problem, a generalisation of his paper was much more difficult. N. N. Bogoluibov proposed to derive hydrodynamic 
equations for superfluid helium 4 describing dissipation to his younger coworkers, but because of the already planned holidays with families they suggested, what was so fortunate for me, to postpone the calculations till the autumn.

In order to seize the occasion and to enter the subject which was new to me it was necessary to repeat all the calculations of Bogoliubov's paper. This helped me a lot in the preparation of the proof.

For me all this was a very happy coincidence. After five years the problems concerning superconductivity had been exhausted and quit unexpectedly I was introduced into a new interesting subject. I prepared and published a paper devoted to the viscous liquid approximation for superfluid Bose systems and many other papers devoted to superfluid Bose systems. I was also interested in the theory of superfluid $\mathrm{He}^{4}$ and $\mathrm{He}^{3}$ and their mixtures. In 1965-66 I was very interested in the hydrodynamic equations for the superfluid Fermi systems. After inspiring the discussion with N. N. Bogoliubov, I introduced to the initial Hamiltonian describing fermions the term containing "sources of particles" analogous to the Bogoliubov "sources of particles" for superfluid Bose systems [6]. This initiative was profitable later when I became interested in the superfluidity of $\mathrm{He}^{3}$. By proper choice of the form of "sources of pairs" I was able to describe all the broken symmetries in the different phases of superfluid $\mathrm{He}^{3}$. The mentioned results exited much interest among specialists and I was looked upon as a student of master Bogoliubov. Therefore, when prof. D. ter Haar proposed me to write the books "Superconductivity and Quantum Fluid" (1970, 237 p.) and "Helium 4" (1971, 336 p.), both edited in Oxford by Pergamon Press, he asked to write them in the spirit of the Bogoliubov School.

For the "papers containing remarkable results in the theory of quantum fluids" I was awarded (with J. Cherwonko) in 1983 the prestigeous Maria Skłodowska-Curie Prize. Our papers were quoted, among others, in ten monographs.

As a senior enjoying good relations with Dubna, I always propagated and supported the scientific contacts with the Laboratory of Theoretical Physics JINR, especially among the theoretical community in Wrocław. In 1987, the President of the Polish Atomic Agency asked me to prepare the materials summarizing the results of our collaboration with JINR in theoretical physics. I prepared a report "Collaboration of the Polish Scientific Institutions with JINR in Theoretical Physics 1956-1986" with the list of all Polish physicists working in the Laboratory of Theoretical Physics JINR. I presented this report at the 25th jubilee session of the Scientific Council of the Laboratory of Theoretical Physics in June 1987.

It is worth while stressing that thanks to the longterm stays of the younger members of our Institute at the Laboratory of Theoretical Physics, directed by prof. N. N. Bogoliubov, 4 doctorates and 6 habilitations were prepared.

In order to honor the contributions of Nikolai Nikolaievich to the scientific exchange with the University of
Wroclaw the Senate of our University awarded him the degree of doctor "Honoris causa" in 1970. During his visit to Wrocław N. N. Bogoliubov was guided, among others, to several historical churches. It happened so that his knowledge about many Bible sceneries exceeded the knowledge of his guides. It is not commonly known that he was the son of an orthodox priest. Unfortunately, in the wake of the revolution, he could not be accepted as the University student, i.e. formally he never completed his university education. N. N. Bogoliubov, born in 1909, joined in Kiev a seminar under Academician N. M. Krylov and in 1930 received his Ph. D. in Mathematics at the Academy of Science. In Dubna I heard a popular joke that Nikolai Nikolaievich, like people without university studies, diminishes the statistics of higher education in JINR.

In Kiev N. N. Bogoliubov lived with a Polish family. Hence he understood Polish and could read in Polish. Therefore, he always asked me to bring as many Polish detective books as possible which he liked very much. The editorial policies of the Moscow circles he considered quite insufficient.

Since I had learned that Nikolai Nikolaievitch liked very much asparagusses I brought him his titbit when my visits to Dubna fell on a proper time. Once I had the opportunity to buy in Wrocław the Bible in Russian, so I "smuggled" it and gave it to Nikolai Nikolaievitch as a gift. He really enjoyed this very much.

N. N. Bogoliubov liked Poland and was very interested in the political and economic situation in our country. When I arrived in Dubna he always found time for scientific discussions and also for news from Poland. He was especially affected after such events as the workers protests in June 1956 in Poznań and in December 1970 in Gdańsk or students protests in March 1968. It was clear that he supported the protesting side.

On the occasion of Nikolai Nikolaievitch's birthday in August conferences in Dubna were organized devoted to the problems of statistical Physics. The organizers invited specialists from abroad and numerous specialists of Bogoliubov's School. Besides scientific sessions, the excursions by boat to the islands on the Moscow Sea were arranged as well as picnics with "buckets" of caviar. Once Bogoliubov's son asked me not to join an excursion because his farther had invited me to a special party. We were transported by a motor boat to a picturesque place. About ten persons, the family and the closest collaborators of Nikolai Nikolaievitch. He was a charming host. I remember delicious Russian fish dishes, excellent wine and cognac (which he considered as an elixir of wisdom).

Just recently (1993) together with prof. C. P. Enz (Geneva University) we payed attention to the very known and important Bogoliubov model (1947) of weakly interacting Bose gas. This exactly solvable model played an important role in the theory of superfluidity and later on in the of theory of superconductivity. Namely, L. D. Landau in his first paper (1941) devoted to superfluidity of $\mathrm{He}^{4}$ assumed that the energy spectrum, for momenta starting from zero, had two branches describing phonons and rotons respectively. From Bogoli- 
ubov's paper (1947) it follows that for momenta close to zero, the energy spectrum has only a phonon branch. After this paper L. D. Landau proposed, also in 1947, his known spectrum for superfluid helium with the roton part "far" from $p=0$.

In the mentioned paper of $1947 \mathrm{~N}$. N. Bogoliubov proposed his famous $(u, v)$ canonical transformation leading to the new practically noninteracting Bose quasiparticles [7]. This type of transformation was generalized by Bogoliubov in 1958 to the fermion systems in order to explain with success the phenomenon of superconductivity [8].

In 1955 M. R. Schafroth showed that the charged free bosons gas exhibits the essential features of the superconducting system. After the discovery of high critical temperature superconductors (1986) one finds that Schafroth model has some properties characteristic of the new type superconductors. Therefore we decided [9] to consider the superconductivity of the charged weakly interacting bose gas. The estimated parameters like penetration depth of the magnetic field and correlation length have perfect order of magnitude from the point of view of $\mathrm{HT}_{c} \mathrm{~S}$.

I had my last contact with N. N. Bogoliubov in 1991. Namely, I was visiting prof. H. W. Capel at the University of Amsterdam and at same time N. N. Bogoliubov (jr.) was there. Being in Amsterdam with my wife and having a car we were sightseeing together with him during all weekends the most attractive places in Holland. Since N. N. Bogoliubov (jr.) was also invited to the University in Antwerp, we decided to give him a ride in order to visit some places in Belgium recommended in the guide books.

When we arrived in Antwerp, Nikolai Nikolaievitch (jr.) phoned his father in Moscow with the information that now he was in Belgium. He reported also with enthusiasm about our recent excursions. Then in a short conversation Nikolai Nikolaievitch thanked me for our helping his son and asked when I would arrived in Dubna. At that time I could not imagine that in half a year $\mathrm{He}$ would pass away.
[1] N. N. Bogoliubov, Usp. Fiz. Nauk 67, 549 (1959).

[2] Z. M. Galasiewicz, Prog. Theor. Phys. 23, 197 (1960).

[3] Z. M. Galasiewicz, Acta Phys. Pol. 19, 467, 495 (1960).

[4] N. N. Bogoliubov, Quasiaverages in problems of statistical physics, Dubna preprint (1961); Lectures on Quantum Statistics (Gordon and Breach, New York, 1970), Vol. 2, Ch. 1.

[5] N. N. Bogoliubov, On hydrodynamics of a superfluid, Dubna preprint (1963); Lectures on Quantum Statistics
(Gordon and Breach, New York, 1970), Vol. 2, Ch. 3.

[6] Z. M. Galasiewicz, Phys. Lett. 15, 39 (1965); Nucl. Phys. 76, 145 (1966).

[7] N. N. Bogoliubov, J. Phys. USSR 11, 23 (1947).

[8] N. N. Bogoliubov, Nuovo Cimento 7, 794 (1958); Zh. Eksp. Toer. Fiz. 34, 58 (1958).

[9] C. P. Enz, Z. M. Galasiewicz, Physica C 214, 239 (1993); see also Z. M. Galasiewicz, M. Wolf, Physica C 248, 49 (1995).

\title{
МОЇ ЗУСТРІЧІ З ПРОФЕСОРОМ М. М. БОГОЛЮБОВИМ У ЛАБОРАТОРІЇ ТЕОРЕТИЧНОЇ ФІЗИКИ ОІЯД У ДУБНІ
}

\author{
3. М. Галасєвіч \\ Інститут фізики Вроилавсъкого технологічного університету, \\ вул. Вибжеже Виспянъскето, 27, 50-370 Врочлав, Полъща
}

Стаття містить спогади про співпрацю та зустрічі з видатним ученим нашого століття — професором М. М. Боголюбовим. 